\title{
XMM-Newton observations of the nitrogen-rich Wolf-Rayet star WR 1
}

\author{
R. Ignace ${ }^{1}$, L. M. Oskinova ${ }^{2}$, and J. C. Brown ${ }^{3}$ \\ 1 Department of Astronomy, University of Wisconsin, Madison, WI 53706, USA \\ 2 Astrophysik, Univerität Potsdam, Am Neuen Palais 10, 14469 Potsdam, Germany \\ 3 Department of Physics and Astronomy, University of Glasgow, Glasgow G12 8QQ, UK
}

Received 14 May 2003 / Accepted 27 June 2003

\begin{abstract}
We present XMM-Newton results for the X-ray spectrum from the N-rich Wolf-Rayet (WR) star WR 1. The EPIC instrument was used to obtain a medium-resolution spectrum. The following features characterize this spectrum: (a) significant emission "bumps" appear that are coincident with the wavelengths of typical strong lines, such as MgXI, SiXIII, and SXV; (b) little emission is detected above $4 \mathrm{keV}$, in contrast to recent reports of a hard component in the stars WR 6 and WR 110 which are of similar subtype; and (c) evidence for sulfur K-edge absorption at about $2.6 \mathrm{keV}$, which could only arise from absorption of X-rays by the ambient stellar wind. The lack of hard emission in our dataset is suggestive that WR 1 may truly be a single star, thus representing the first detailed X-ray spectrum that isolates the WR wind alone (in contrast to colliding wind zones). Although the properties of the S-edge are not well-constrained by our data, it does appear to be real, and its detection indicates that at least some of the hot gas in WR 1 must reside interior to the radius of optical depth unity for the total absorptive opacity at the energy of the edge.
\end{abstract}

Key words. stars: individual: WR 1 (HD 4004) - stars: winds, outflows - stars: early-type - X-rays: stars

\section{Introduction}

The XMM-Newton and Chandra X-ray satellites are providing high resolution X-ray spectra of hot stars that challenge our understanding of winds from early-type stars in new and interesting ways. The X-ray luminosities $L_{X}$ of OB stars are a small fraction of their Bolometric $L_{*}$ output, commonly at the level of about $10^{-7}$ by fraction (e.g., Seward et al. 1979; Berghöfer et al. 1997), or even smaller for the later B stars (Cohen et al. 1997). Yet, the X-ray emission is interesting because it is telling of processes that generate hot gas in the winds or atmospheres of single hot stars, with wind shocks having generally been the favored candidate for understanding the presence of hot gas (e.g., Lucy \& White 1980; Lucy 1982).

However, high spectral resolution data are revealing that the observed line profile shapes are different from the model predictions. For example, resolved X-ray lines in $\mathrm{O}$ and B stars often appear symmetric (e.g., Schulz et al. 2000; Waldron \& Cassinelli 2001; Miller et al. 2002; Cohen et al. 2003), whereas basic approaches to modeling distributed wind-shocks predict asymmetric profile shapes (e.g., MacFarlane et al. 1991; Ignace 2001; Owocki \& Cohen 2001; Ignace \& Gayley 2002; Feldmeier et al. 2003). Even though most of the emission is dominated by soft $\mathrm{X}$-rays $(\lesssim 1 \mathrm{keV})$ as expected from wind

Send offprint requests to: $\mathrm{R}$. Ignace,

e-mail: ignace@astro.wisc.edu shocks (e.g., Feldmeier et al. 1997), a significant and unexpected harder component is also seen (e.g., Waldron \& Cassinelli 2001; Skinner et al. 2002a,b).

However, relatively few hot stars have been observed with the latest generation instruments, and the interpretations are complicated by several factors. Of the $\mathrm{O}$ stars observed with Chandra, $\zeta$ Pup is the only source to show X-ray emission profiles that are consistent with theoretical expectations for distributed wind shocks (Cassinelli et al. 2001; Kramer et al. 2003; Feldmeier et al. 2003). The stars $\zeta$ Ori, $\delta$ Ori, $\theta^{1}$ Ori C, and $\tau$ Sco all display unshifted and symmetric (and in some cases narrow) emission line profiles. It would seem then that the standard wind-shock model is now suspect; however, the observational selection effects must be carefully assessed. All of these stars were selected based on their being relatively $\mathrm{X}$-ray bright. It turns out that two of these systems are binaries, one is known to be a magnetic star with surface fields in excess of 1 kiloGauss ( $\theta^{1}$ Ori C - Donati et al. 2002), and one shows peculiar UV P Cygni lines suggesting infalling clumps of gas (Howk et al. 2000). A larger observational sample is clearly needed to determine the status of the wind-shock paradigm for understanding OB star X-ray emissions.

This paper reports on XMM-Newton observations of a single star of the Wolf-Rayet class. The Wolf-Rayet stars are the evolved counterparts (as evidenced by being H-deficient and metal-enriched) of massive $\mathrm{O}$ stars with initial masses in 
excess of about $20 M_{\odot}$. The main advantage of selecting single WR stars for X-ray studies is that their mass-loss rates are an order of magnitude greater than their $\mathrm{O}$ star progenitors. This means that the winds are considerably denser, the photoabsorption by the ambient wind of shock-generated X-rays is more severe, and one can be fairly certain that the soft X-rays can escape only from large radii (at $1 \mathrm{keV}, 100$ 's or even 1000's of $R_{*}$ ). The wind absorption, primarily bound-free HeII and $\mathrm{K}$-shell absorption of metals, is a strong function of energy such that it is much less at higher X-ray energies, and so hard $\mathrm{X}$-ray emission can emerge from much deeper depths in the wind flow. Still, in the soft band the X-rays must come from large radius, and the major benefit of this is that the wind can be assumed to be in constant expansion. One may expect a roughly steady-state scenario, which can be understood as follows. The wind flow time is characterized by $R_{*} / v_{\infty} \approx$ hours. Defining $r_{1}$ to be the radius at which the photo-absorptive optical depth is unity at some energy $E$, the fact that $r_{1} / R_{*} \gg 1$ means that the crossing time $r_{1} / v_{\infty}$ is much longer than the characteristic wind flow time. Consequently, one can expect that whatever structures give rise to the X-ray emission will be "numerous" in the interval $1 r_{1}$ to $2 r_{1}$, hence the emission properties may be approximated as nearly stationary. And so one might expect that the X-ray spectra of single stars with dense winds should be straightforward to interpret.

That is the expectation, but recent observations have revealed some interesting surprises. Oskinova et al. (2003) have reported on observations of the carbon-rich star WR 114. For a long exposure of $16000 \mathrm{~s}$, no X-rays were detected in the EPIC 0.2-10.0 keV band, although a well-resolved X-ray spectral shape was expected. Oskinova et al. set an upper limit to the $\mathrm{X}$-ray luminosity of $L_{\mathrm{X}}<4 \times 10^{-9} L_{*}$, that is low compared to $\mathrm{O}$ stars. Indeed, the authors investigated existing pointed and serendipitous observations of WC stars from a variety of X-ray satellites and concluded that no single WC star has been definitively detected in the X-ray band. They attribute the lack of $\mathrm{X}$-rays to strong wind absorption in these dense and highly metal-enriched (especially carbon) stellar winds. (Although one cannot rule out the possibility that the production of X-rays in WC stars is physically different from what occurs in O stars, and that this difference explains the low X-ray luminosities in WC stars.)

Another surprise comes from XMM-Newton observations presented in Skinner et al. (2002a,b) for two nitrogen-rich WR stars (WN) - WR 6 (=EZ CMa = HD 50698) and WR 110. Both of these are believed to be single stars (although there has been vigorous debate over WR 6 owing to the well-known periodicity of 3.765 days). Yet, the XMM-Newton spectra show a hard component, which if thermal would suggest the presence of 30-40 million degree gas. Although no detailed hydrodynamic simulation has been made for wind shocks in a WR stellar wind, Gayley \& Owocki (1995) have considered the line-driven instability mechanism in the context of the dense WR winds, and conclude that the production of shocks and wind structuring similar to that in the $\mathrm{O}$ stars should occur. But large amounts of hot gas at around $30+$ million $\mathrm{K}$ is not predicted in O star simulations. Skinner et al. (2002a) indicate that magnetic wind confinement could produce hot gas of these high
Table 1. Stellar parameters for WR 1.

\begin{tabular}{lr}
\hline \hline Parameter & Value \\
\hline Distance $d$ & $1.82 \mathrm{kpc}$ \\
Effective temperature $T_{*}$ & $100000 \mathrm{~K}$ \\
Luminosity $\log L_{*}$ & $5.3 L_{\odot}$ \\
Mass-loss rate $\dot{M}$ & $10^{-4.834} M_{\odot} \mathrm{yr}^{-1}$ \\
Terminal velocity $v_{\infty}$ & $1600 \mathrm{~km} \mathrm{~s}^{-1}$ \\
Density contrast $D$ & 4.00 \\
Filling factor & 0.25 \\
Relative abundances & $($ by number) \\
$\mathrm{He}$ & 1.00 \\
$\mathrm{~N}$ & $4.36 \times 10^{-3}$ \\
$\mathrm{C}$ & $6.68 \times 10^{-5}$ \\
$\mathrm{Fe}+\mathrm{Co}+\mathrm{Ni}$ & $1.02 \times 10^{-4}$ \\
\hline
\end{tabular}

temperatures, but the implied surface fields would be quite high at several kiloGauss, and so they prefer wind collision onto a non-degenerate companion.

We present an analysis of XMM-Newton observations of a third WN star, WR 1, in an attempt to clarify the nature of $\mathrm{X}$-rays from this class of evolved massive stars. Basic stellar and wind parameters for WR 1 are listed in Table 1, as derived from model fitting of the UV/optical spectrum using a grid of WN star models that includes iron blanketing (Hamann, private comm.). Important to this work is the determination of stellar abundances and the interstellar column density, because K-shell photo-absorption by both the wind and the ISM affects the source spectrum that we observe at the Earth. For WR 1 , a color excess of $E_{B-V}=0.8$ is derived by nulling the $2200 \AA$ interstellar feature. Using Groenewegen \& Lamers (1989), we adopt the relation $N_{\mathrm{H}}=(3.8 \pm 0.9) \times 10^{21} E_{B-V} \mathrm{~cm}^{-2}$ to obtain an interstellar column density of neutral hydrogen of $N_{\mathrm{H}}=(3.04 \pm 0.7) \times 10^{21} \mathrm{~cm}^{-2}$ toward WR 1 .

We adopt these source and interstellar parameters for use in our analysis of the X-ray data. The observations and their reduction are described in Sect. 2. A discussion of the results is presented in Sect. 3, especially against the backdrop of results for WR 6 and 110. Finally in Sect. 4, we draw conclusions about the state of X-rays from WR winds and future observations needed to increase our understanding of these flows.

\section{XMM-Newton observations of WR 1}

\subsection{The X-ray data}

XMM-Newton observed WR 1 on 21 August 2002 for $\approx 9.2 \mathrm{ksec}$ in total, with a total flux of $9.7 \pm 0.8 \times$ $10^{-14} \mathrm{erg} \mathrm{cm}^{-2} \mathrm{~s}^{-1}$ in the $0.2-10.0 \mathrm{keV}$ EPIC passband (see Table 2). Technical details regarding the XMM-Newton observatory and its performance are described in a special letters issue of A\&A (vol. 365). Data were obtained with the European Photon Imaging Camera (EPIC). EPIC is equipped with two identical MOS cameras and a PN camera. These provide CCD imaging spectroscopy with energy coverage between $0.2-15 \mathrm{keV}$ in a 30 arcmin diameter field of view (FoV) with an angular resolving power of approximately $12 \operatorname{arcsec} F W H M$. The spectral resolving power of EPIC ranges between 
Table 2. XMM-Newton observations of WR 1.

\begin{tabular}{ll}
\hline \hline Parameter & Value \\
\hline Start [UT] & $2002-08-2023: 50$ \\
End [UT] & $2002-08-2102: 47$ \\
Exposure [ksec] & $9.2(\mathrm{MOS}), 7.6(\mathrm{PN})$ \\
Count Rate $\left[10^{-2} \mathrm{cnt} \mathrm{s}^{-1}\right]$ & \\
\multicolumn{1}{c}{ MOS1 } & $9.03 \pm 0.37$ \\
MOS2 & $9.06 \pm 0.36$ \\
PN & $20.94 \pm 1.08$ \\
\hline
\end{tabular}

$E / \Delta E=30$ at $0.1 \mathrm{keV}$ to about 200 at $10 \mathrm{keV}$. Thus the resolution is about 100 for most of the spectrum, which although inadequate for resolving the wind-broadened lines in hot star spectra, does provide an excellent spectral shape for the energy distribution in the X-ray band, which is a major advance over previous missions that have yielded only passband fluxes for single WR stars (e.g., Pollock 1987; Pollock et al. 1995).

Observations were made using the full-window mode and the medium blocking filter. After excluding contaminated time intervals due chiefly to soft proton flares, the useful exposure time dropped to $7.9 \mathrm{ksec}$ for MOS1, $8.4 \mathrm{ksec}$ for MOS2, and $6.1 \mathrm{ksec}$ for the PN instrument. The X-ray position obtained by averaging the results of all three EPIC cameras is RA(2000) = $00^{\mathrm{h}} 43^{\mathrm{m}} 28^{\mathrm{s}} .34$ and $\operatorname{Dec}(2000)=64^{\circ} 45^{\prime} 35^{\prime \prime}$. 5 , with an offset of a mere 0.'4 from the optical position (van der Hucht 2001), providing high confidence that the X-ray source is in fact WR 1 .

\subsubsection{X-ray images}

Data reduction followed standard procedures using the XMMNewton Science Analysis Software (SAS vers. 5.3.3). The latest calibration files were used, and data were filtered to select $\mathrm{X}$-ray related events. Spectra and light curves were accumulated from the filtered event lists over a circular region of radius $\approx 40$.'0. Data from MOS1 and MOS2 were combined to increase the signal-to-noise ratio $(\mathrm{S} / \mathrm{N})$.

The broad-band $(0.2-10 \mathrm{keV}) \mathrm{PN}$ image yields a total of 1482 counts from WR 1 in $6.1 \mathrm{ksec}$, while MOS1 gives 783 counts in $7.9 \mathrm{ksec}$ and MOS2 gives 825 counts in $8.4 \mathrm{ksec}$. There are 70 counts above $3 \mathrm{keV}$ combined in the MOS 1 and MOS 2 detectors, and 60 counts for the PN. WR 1 was also observed by the RosAT PSPC (0.2-2.4 keV) with $33.8 \mathrm{ksec}$ of exposure time. The detected count rate was $0.0335 \pm 0.0012 \mathrm{cps}$, in good agreement with our XMM-Newton observations after correcting for differences in the effective areas. Using an annulus centered on the source, we obtained a background spectrum from our XMM-Newton images. The background level was determined to be $\approx 0.0023 \mathrm{cps}$ for each of the MOS instruments and $\approx 0.005$ cps for PN.

Figure 1 shows the inner region of the MOS1 image within $\approx 3$ arcmin of the target. The different panels are for different energy bands, with the full band width $(0.2-10.0 \mathrm{keV})$ shown at upper left, and narrower energy bands shown as indicated. The star is well-detected up to $4 \mathrm{keV}$. The final panel at lower right is for the band $4-10 \mathrm{keV}$ showing that there is no discernable source at these energies. This is fascinating because hard

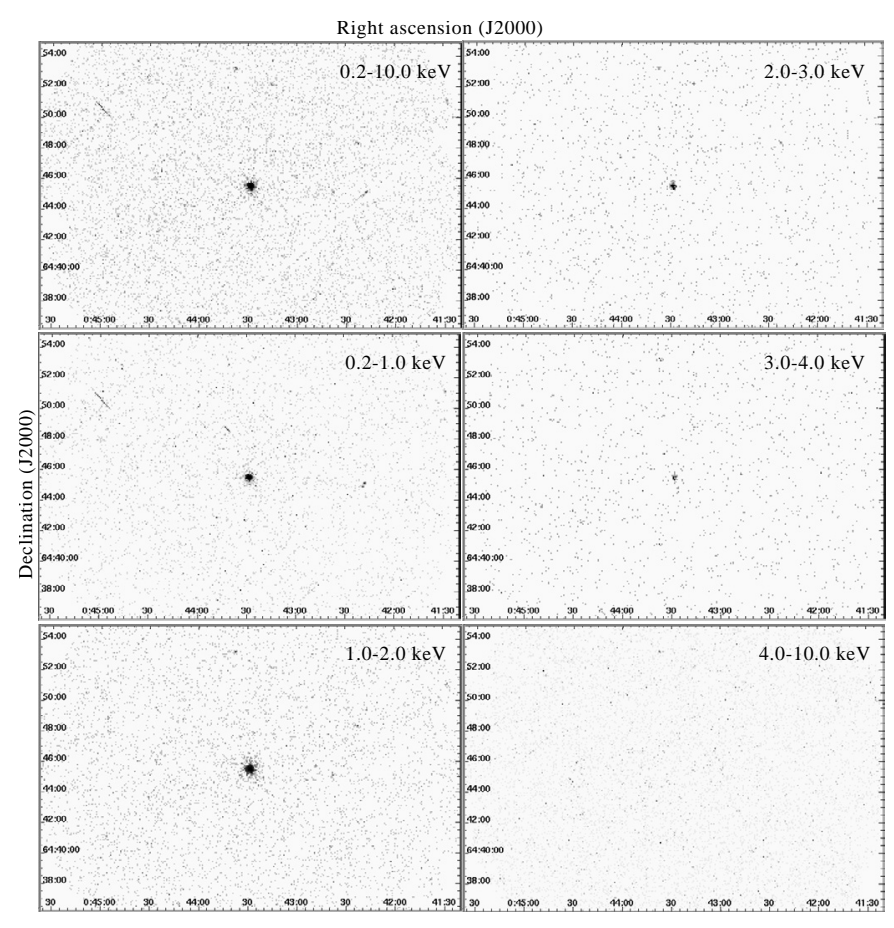

Fig. 1. The inner region of the MOS1 image of WR 1 in different energy bands as indicated in the upper right corner of each panel. It is clearly seen that the bulk of emitted X-ray photons has energies between $0.2-2 \mathrm{keV}$. The star is clearly detectable up to $4 \mathrm{keV}$, but not at higher energies.

emission has been detected in two other WR stars of similar types (Skinner et al. 2002a,b). Further comments on the implications of hard band emission will be made later.

\subsubsection{X-ray light curves and timing analysis}

WR 1 shows variability in its optical spectrum, which can be significant in the equivalent widths of some emission lines; however, Niedzielski (2000) does not find any periodic variability in the range of $30 \mathrm{~min}$ to 2 days, although there is some indication that a longer period may be present. Using the 1992-93 observation of WR 1 with ROSAT and our XMMNewton data, we have produced a light curves and find no evidence for X-ray variability in either dataset at the $10 \%$ level or above with a sampling time of about $6 \mathrm{~min}$.

\subsubsection{X-ray spectra}

The spectrum obtained with the EPIC-PN is shown in Fig. 2. Although unresolved, several emission lines reveal their presence as "bump" features, including MgXI, SiXIII, and likely SxV. The crowded region around $1 \mathrm{keV}$ is probably due to numerous lines of FeXVII, by analogy with the resolved X-ray spectra from O stars (e.g., Kahn et al. 2001; Waldron \& Cassinelli 2001; Cassinelli et al. 2001). Of special interest is the suggestive presence of NVI line emission at $0.43 \mathrm{keV}$ and NVII at $0.50 \mathrm{keV}$.

The X-ray spectra from the EPIC instruments were analyzed with XSPEC vers. 11.2 using a variety of models including 


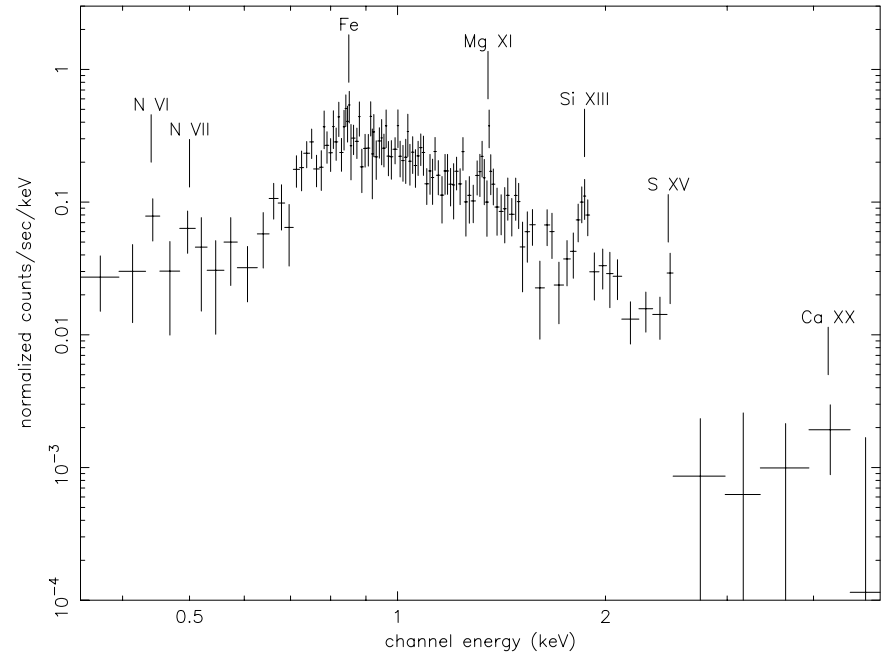

Fig. 2. Background-subtracted EPIC-PN spectrum of WR 1 (WN4) corrected for the instrumental area sensitivity and binned to a minimum of 10 counts per bin. "Normalized" is defined such that when multiplying the vertical axis by the useful exposure time, one obtains the total counts in each bin as represented by the plotted points. Shown are the location of several commonly observed strong X-ray lines. The label "Fe" indicates the general vicinity of several lines of different highly ionized iron species.

discrete temperature approximations (VMEKAL, VAPEC) and multi-temperature models for differential emission measure (C6PVMEKAL). For the harder emission, nonthermal powerlaw models were also examined. Our analysis, based on rather simple models, is meant to characterize the overall spectral shape. We do not claim that the models accurately describe the physical conditions in the emitting plasma, and the best fit parameters should therefore not be overinterpreted. The most prominent flaw of the spectral modeling is that the absorption of X-rays in the WR wind (warm absorption) is treated only in an approximate way, in which absorption edges only are directly attributed to the ionized wind. What prevents us from applying a proper wind absorption model is ignorance regarding the X-ray generation by the winds of WR stars in conjunction with the modest resolution of our dataset. With only spectral shape information, it is difficult to distinguish between warm absorption by the wind from cold absorption by the ISM, given that the emissivity model is not well-constrained.

We found that the best fits obtained for the spectra from all three EPIC instruments are quite similar, and so hereafter we focus on results obtained for a simultaneous fit to the data from all of the EPIC instruments. We considered a number of models for the thermal collisionally-ionized optically thin diffuse gas to account for the emission. The interstellar absorption was required to have an column density not smaller than the interstellar HI value, as obtained from the UV spectral analysis of WR 1.

Initially, solar-metalicity MEKAL models were considered (Mewe et al. 1985; Kaastra 1992), but neither one-temperature nor two-temperature models provide an adequate fit to the data. This is not surprising since the assumption of solar abundances is known a priori to be false from the UV/optical analysis. Consequently, we adopted a chemical composition

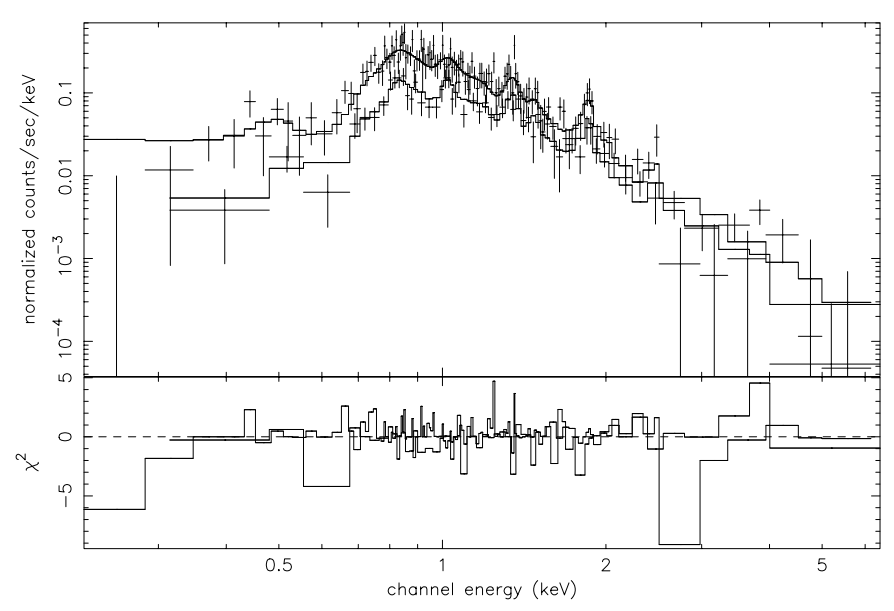

Fig. 3. EPIC MOS1 and PN spectra folded with a $2 T$ VAPEC model $\left(k T_{1}=0.6 \mathrm{keV}, k T_{2}=2.5 \mathrm{keV}\right)$ using $\mathrm{WN}$ abundances. The lower panel represents reduced $\chi^{2}$. Although the overall statistic is acceptable at $\chi^{2} /$ d.o.f. $=0.87$, it is obvious that the fitting to prominent spectral features is poor.

appropriate for WR 1 as given in Table 1, consisting of mainly $\mathrm{He}$, with $\mathrm{N}$ enhanced, $\mathrm{C}$ and $\mathrm{O}$ both diminished, and otherwise solar proportions for the heavier metals.

Given the modest quality of the data and the rather vast range of fitting parameters, there are many different models yield suitable fits. It turns out that VAPEC models provide somewhat better fits than the VMEKAL ones. The spectra are wellmatched, within the errors, using combinations of one, two, and three temperature models along with altered abundances, and variable interstellar absorption. Therefore, we decided to choose among the models with similar statistics those that most accurately represent the prominent spectral features and yet provide an adequate physical interpretation.

At first we concentrated on those models that require a level of absorption that is consistent with the known ISM value alone (i.e., no warm absorption from the wind). A one-temperature model with a WN chemical composition yields a poor fit that fails to match prominent spectral features. The twotemperature models are better; however, extraordinarily high $\mathrm{N}$ abundances are required when the cold absorption is fixed at the interstellar value given by the UV spectral modeling.

Allowing both the hydrogen column density $N_{\mathrm{H}}$ and the abundances to vary, Fig. 3 shows our best two-temperature model fit. The model parameters are given in Table 3, including the goodness of fit $\chi^{2} /$ d.o.f., the two temperature ( $2 T$ ) components, the sulfur absorption edge properties (see below), the $\mathrm{Fe}$ abundance (relative to solar), $K_{1}$ and $K_{2}$ are model normalization coefficients for the respective two temperature components, and finally the model X-ray flux $F_{\mathrm{X}}$ in the EPIC energy passband of $0.2-8.0 \mathrm{keV}$. The coefficients $K_{1}$ and $K_{2}$ correspond to $10^{-14}\left(4 \pi d^{2}\right)^{-1} \int n_{\mathrm{e}} n_{\mathrm{i}} \mathrm{d} V$, where $d, n_{\mathrm{e}}$, and $n_{\mathrm{i}}$ are respectively the distance (in $\mathrm{cm}$ ) of WR 1 , and the electron and ion densities $\left(\mathrm{in}^{-3}\right.$ ) of the X-ray emitting plasma.

Assuming absorption by the ISM only, the first column of Table 3 gives the fitting parameters. The iron abundance (by number) is treated as variable and is nearly solar for this fit. The interstellar column density required to match the X-ray 
Table 3. Best fit models.

\begin{tabular}{lcc}
\hline \hline Model & $\begin{array}{c}2 T \text { Thermal, } \\
\text { ISM absorption only }\end{array}$ & $\begin{array}{c}2 T \text { Thermal } \\
\text { ISM and wind absorption }\end{array}$ \\
Parameters & & \\
\hline$\chi^{2} /$ d.o.f. & 0.98 & 0.66 \\
$k T_{1}[\mathrm{keV}]$ & $0.56-0.67$ & $0.57-0.63$ \\
$k T_{2}[\mathrm{keV}]$ & $1.27-3.20$ & $\leq 2.1$ \\
$k T_{\text {edge }}[\mathrm{keV}]$ & - & $2.5-2.6$ \\
$\tau_{\text {edge }}$ & - & $2.3-10.0$ \\
$\mathrm{Fe} / \mathrm{Fe}_{\odot}$ & $0.9-1.7$ & $1.8-4.9$ \\
$K_{1}\left[\mathrm{~cm}^{-3}\right]$ & $8 \times 10^{-8}$ & $5.6 \times 10^{-8}$ \\
$K_{2}\left[\mathrm{~cm}^{-3}\right]$ & $4 \times 10^{-8}$ & $5.0 \times 10^{-8}$ \\
$N_{\mathrm{H}}\left[10^{22} \mathrm{~cm}^{-2}\right]$ & $0.38-0.51$ & $0.27-0.32$ \\
$F_{\mathrm{x}}^{a}\left[10^{-13} \mathrm{erg} \mathrm{cm}^{-2} \mathrm{~s}^{-1}\right]$ & 4.9 & 5.2 \\
\hline
\end{tabular}

${ }^{a}$ Integrated fluxes in the $0.2-8.0 \mathrm{keV}$ energy band.

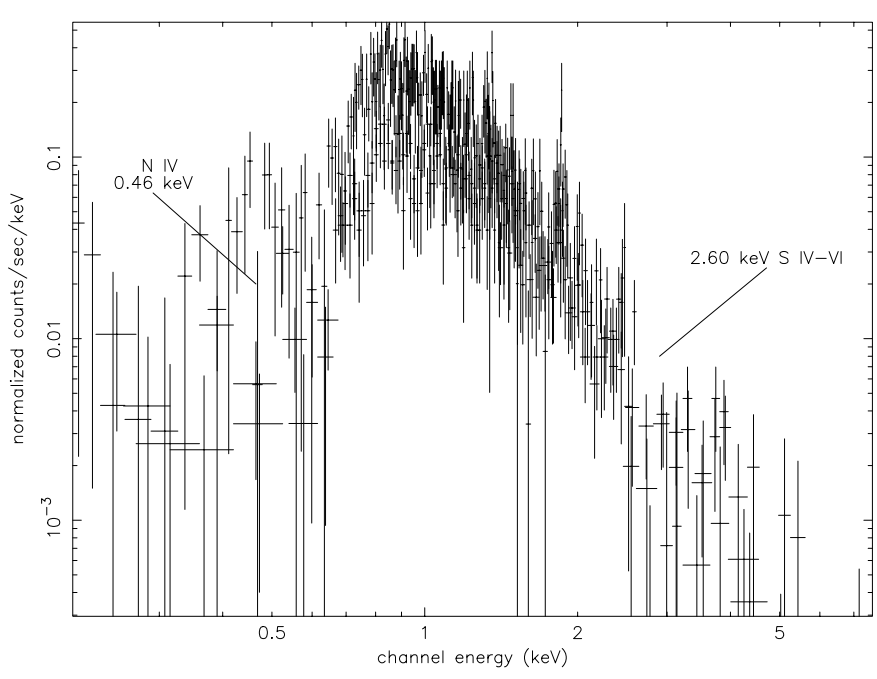

Fig. 4. MOS1, MOS2, and PN spectra rebinned to contain at least 5 counts per bin. Although the data are noisy, the absorption gaps at about $0.46 \mathrm{keV}$ and especially at about $2.56 \mathrm{keV}$ are clearly seen. The K-shell absorption edges of NIV and SIV-VI are located at those energies (Daltabuit \& Cox 1972).

spectrum of WR 1 is within a factor of 2 of that derived from the UV modeling. Importantly, the behavior of the $\chi^{2}$ shown in the lower panel of Fig. 3 highlights problems with reproducing prominent spectral features, notably the nitrogen and calcium bumps at around $0.4 \mathrm{keV}$ and $4.2 \mathrm{keV}$ respectively, and a couple of drop-outs in flux, most significantly at $0.6 \mathrm{keV}$ and $2.6 \mathrm{keV}$. Based on the discrepancy in the $\mathrm{N}$ and $\mathrm{Fe}$ abundances and the overall poor spectral shape representation, we choose to reject this model.

We found that our modeling efforts consistently failed to reproduce the spectrum around $0.6 \mathrm{keV}$ and $2.6 \mathrm{keV}$, energies that coincide with K-shell photo-ionisation edges of NVI and SIV-VI, respectively. These ion states should be the dominant stages for each respective atomic species in the wind of WR 1 (Hamann, private comm.). In Fig. 4, the spectra for MOS1, MOS2, and PN are overplotted at a minimum 5 counts per bin.

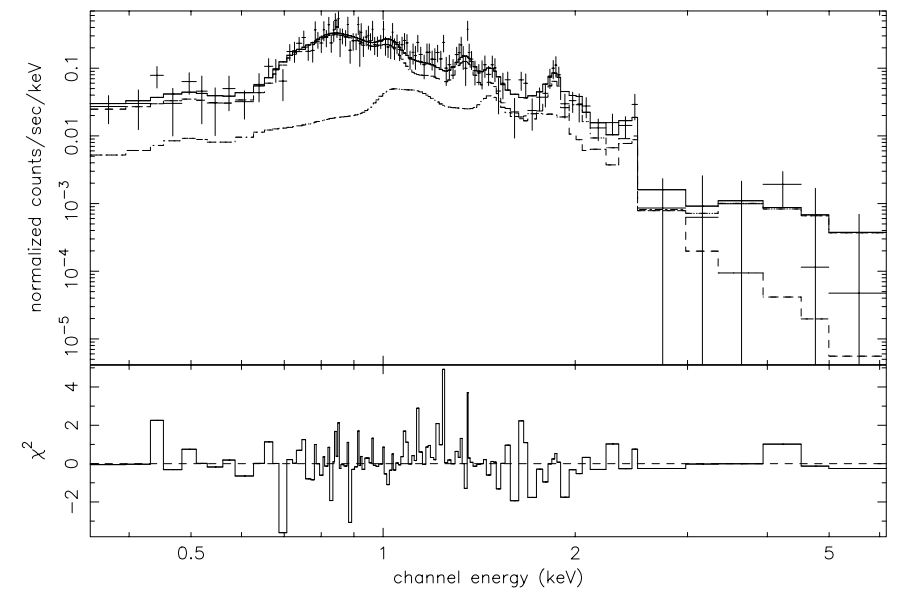

Fig. 5. Two temperature fit with fixed WR 1 abundances and SIV edge by the stellar wind. The fitting statistic is extremely good (the null hypothesis probability is 0.997 ), and the all prominent spectral features are reproduced. Contributions from "cold" and "hot" plasmas are shown. Parameters for the model are listed in the last column of Table 3.

The two absorption features are seen in all three independent detectors and cannot therefore be explained by instrumental effects. The data are somewhat noisy and the resolution smears out the drop across the edge, so that we must content ourselves with claiming a tentative detection of these edges that need to be confirmed with a longer exposure and better spectral resolution.

The feature near $2.6 \mathrm{keV}$ is particularly interesting since the data are adequate for deriving an optical depth, with a value of $\tau_{\text {edge }} \approx 6$. This absorption can only arise from the wind, because the optical depth of the interstellar medium at this energy is only about 0.01 . We note that the effective area for the PN instrument shows a drop at around $2.5 \mathrm{keV}$; however, we find the absorption feature in all three of the EPIC instruments, and so we believe that it is likely real. Figure 5 shows the PN spectrum with a model fit that accounts for the stellar wind absorption by sulfur. Model parameters are given in the last column of Table 3 that includes both cold absorption by the ISM and 
warm absorption by the wind to account for the S-edge. The fit is superb, as can be seen from the residuals and the value of the $\chi^{2} /$ d.o.f. statistic. Interestingly, warm absorption by the stellar wind is clearly needed only for the S-edge. The twotemperature model has 0.6 and $2.5 \mathrm{keV}$ components, and we find that a good fit is achieved assuming the edge absorption for either component, or for both of them. Thus we can speculate that the two different temperature plasmas are more or less located in the same region of the stellar wind.

\section{Discussion}

The question before us is how to make the best use of the spectral data to infer the physical properties of the X-ray emitting gas and its implications for the structure of the wind. The simplest realistic model for the X-ray emission, motivated by $1 \mathrm{D}$ radiative hydrodynamic simulation for $\mathrm{O}$ stars (e.g., Owocki et al. 1988; Cooper \& Owocki 1994; Feldmeier et al. 1997), assumes spherical symmetry with thoroughly mixed hot (X-ray emitting) and warm (bulk wind) components (e.g., Baum et al. 1992; Owocki \& Cohen 1999; Ignace et al. 2000), so that the observed X-ray luminosity as a function of energy $E$ is

$L_{\mathrm{X}}(E)=\left[\int j(E, r) \mathrm{e}^{-\tau_{\mathrm{w}}(E, p, z)} \mathrm{d} V\right] \mathrm{e}^{-\tau_{\mathrm{ISM}}(E)}$,

where $p$ and $z$ are cylindrical coordinates, $r$ is the spherical radius, and $\mathrm{d} V$ is a differential wind volume element. The factors appearing in this equation are $j$ the emissivity for the hot gas, $\tau_{\mathrm{w}}$ the optical depth of wind absorption (i.e., warm absorption), and $\tau_{\text {ISM }}$ the absorption by the intervening ISM (i.e., cool absorption). The emergent X-ray luminosity from the source as a function of energy is thus a volume integral over the entire wind, assuming the X-ray emission to be optically thin. The star itself is taken not to be a source of X-rays (i.e., no corona or flares). Of course, it is not the X-ray luminosity spectrum that is actually observed, but rather the brightness spectrum, which includes the effect of the instrumental sensitivity. Consequently, the observed flux of emission (such as appears in our figures) measured by an X-ray sensitive detector with effective collecting area $A_{\text {eff }}(E)$ becomes

$F_{\mathrm{X}}(E)=\frac{L_{\mathrm{X}}(E)}{4 \pi d^{2}} \times \frac{A_{\mathrm{eff}}(E)}{A_{\mathrm{tot}}}$,

where $A_{\text {tot }}$ is the energy-integrated total area, and $d$ is the distance to the star. Actually, Eq. (2) should formally be a convolution involving the spectral smearing of the instrument, but we ignore that fact for the purpose of a qualitative discussion, especially in light of the relatively good spectral resolution of current X-ray instrumentation.

In modeling the observed X-ray spectrum, it has become standard practice to introduce a volume filling factor for the $\mathrm{X}$-ray emitting gas; however, in principle one should take into account the detailed wind-structuring along with the fact that there can be a broad range of hot gas temperatures, and that even the temperature range may vary from one location to the next. Moreover, one would like to allow for variable abundances, using the model dependence on abundances both in terms of the line spectrum and warm absorption (primarily by $\mathrm{K}$-shell absorption) to determine the wind metallicity. Clearly, the volume integration of Eq. (1) over the entire wind represents a strongly convoluted quantity, suggesting that "simplified" models for the wind X-ray emission may yield reasonable fits to the observations, even though such models might not accurately reflect the true physical structure of the stellar flows.

This is a challenging inverse problem (see Craig \& Brown 1986 for technical details regarding inverse methods for astrophysical applications). Even so, based on the EPIC spectrum, we can certainly make several positive comments regarding the $\mathrm{X}$-rays from WR 1 . In particular, the X-ray spectrum of WR 1, up to about $3 \mathrm{keV}$, is basically similar in shape to those observed in WR 6 and WR 110. Indeed, the soft component is characterized by a gas temperature of $k T \approx 0.5-0.6$ for all three stars. Moreover, the low energy emission is largely absorbed by the ISM; peak emission occurs around $1 \mathrm{keV}$, and there is declining emission toward higher energies. Emission features appear at energies that correspond to known strong lines, such as MgXI, SiXIII, and SXV, which are to be expected in analogy with such lines being identified in the high resolution X-ray spectra of OB stars.

We adopt a conventional approach to analyzing the spectrum, using VAPEC models and allowing for variable abundances and ISM absorption column densities. As noted before, parameters for our best fit models of the WR 1 X-ray spectrum appear in Table 3. The X-ray spectral shape data that we have obtained, in conjunction with previously published reports for WR 6 and WR 110 from Skinner et al. (2002a,b), is a significant forward step in the study of winds from single WR stars. It is our opinion that, eventually, resolved X-ray emission lines from single WR stars will be critical for better constraining the modeling effort. Still, the basic properties of the X-ray spectrum yield important information about the X-rays from WR winds, and specifically, we highlight the importance of the possible presence of an absorption edge of ionized $\mathrm{S}$ and the hard emission characteristics of WR 1.

\subsection{Sulfur absorption edge}

If real, the appearance of the S-edge is exciting indeed. For our best fit $2 T$ model, we infer a $90 \%$ confidence range for the edge optical depth of $\tau \approx 2-10$. The jump in optical depth owing to the S-edge by the ISM is expected to be only about 0.01 , much smaller than the inferred value.

Assuming the edge to be real, and a consequence of wind absorption, we can compare the measured value to expectations based on the a priori adopted wind parameters for WR 1 . The influence of the S-edge is to increase discontinuously the total photo-absorption cross-section $\sigma_{\mathrm{w}}$ at the location of the edge itself. It is important to relate the drop in the spectrum to the wind parameters in such a way as to test the underlying model for the X-ray emission. The simplest such model is based on distributed hot gas embedded in a warm absorbing wind. In this context the increase in wind absorption that occurs in crossing the edge means that the hot gas emission measure drops, 
and the observed flux of X-rays drops too, as long as the X-ray emissivity does not vary rapidly across the edge.

The observable is a drop in the X-ray source brightness in moving across the edge from low to high energies. Our goal is to relate this measured decrease to properties intrinsic to the wind. In so doing, allowance can be made for a multitemperature gas, and for simplicity, we assume that the range of hot gas temperatures is uniform throughout the flow. Then the $\mathrm{X}$-ray luminosity produced at any given energy in the spectrum is given by

$L_{\mathrm{X}}(E)=\int n_{\mathrm{X}}^{2} \Lambda_{\text {tot }}(E) \mathrm{e}^{-\tau_{\mathrm{w}}(E, r)} \mathrm{d} V$,

where $n_{\mathrm{X}}$ is the number density for the hot plasma, $\Lambda_{\text {tot }}$ is the total cooling function for the $2 T$ hot plasma (taken to be a constant with radius), and the integral is over the emitting volume. Now the X-ray spectrum measured at the Earth is further affected by the interstellar absorption, but this will not be relevant to the discussion at hand, because this absorption just below and just above the edge is the same.

We introduce the notation $<$ and $>$ for properties just below or above the S-edge, with $L_{<}, L_{>}, \tau_{<}$, and $\tau_{>}$. Hence we have that $\tau_{>}=\tau_{<}+\tau_{\mathrm{S}}$, for $\tau_{\mathrm{S}}$ the optical depth of the S-edge contribution alone.

It has been our assertion that in the standard wind-shock model, the observed X-ray emission must emerge from large radii, owing to the severe photoabsorption by the dense WR wind. Assuming this to be the case, and that the wind is in constant expansion, Eq. (3) can be evaluated analytically for the radius integration analogous to the approach of Ignace (2001). For this case the wind optical depth is

$\tau_{\mathrm{w}}(E, r)=\tau_{\text {tot }}(E) \frac{R_{*}}{r} \frac{\theta}{\sin \theta}$,

where $\theta$ is the polar spherical angle from the line-of-sight, and $\tau_{\text {tot }}$ is the total line-of-sight photo-absorptive optical depth of the wind to the stellar surface at $R_{*}$. Using a change of variable $u=R_{*} / r$, and noting that $\mathrm{d} V=2 \pi r^{2} \mathrm{~d} r \sin \theta \mathrm{d} \theta$ and $n_{\mathrm{X}} \propto r^{-2}$, the X-ray luminosity becomes

$L_{\mathrm{X}}(E) \propto \Lambda(E) \tau_{\text {tot }}^{-1}(E)$.

If we may assume that $\Lambda\left(E_{<}\right) \approx \Lambda\left(E_{>}\right)$, then across the S-edge, we have that

$\frac{L_{<}}{L_{>}}=\frac{\tau_{>}}{\tau_{<}}=1+\frac{\tau_{\mathrm{S}}}{\tau_{<}}$.

The optical depth of the S-edge is given by

$\tau_{\mathrm{S}}=n_{0} \mathcal{A}_{\mathrm{S}} \sigma_{\mathrm{S}} R_{*}$,

where $n_{0}$ is the number density at the base of the wind (related to $\dot{M}, v_{\infty}$, etc.), $\mathcal{A}_{\mathrm{S}}$ is the relative abundance by number of sulfur, and $\sigma_{\mathrm{S}}$ is the photoabsorptive cross-section of the S-edge.

So the ratio of Eq. (8) may be re-expressed as

$\frac{L_{<}}{L_{>}}=1+\frac{\mathcal{A}_{\mathrm{S}} \sigma_{\mathrm{S}}}{\sigma_{<}}$.

We thus have a choice. The edge cross-section for SIV is a laboratory-measured quantity, given by $\sigma_{\mathrm{S}}=1.14 \times 10^{-19} \mathrm{~cm}^{2}$
Table 4. Hard emission from WN stars.

\begin{tabular}{lcccc}
\hline \hline Star & $\begin{array}{c}N_{\mathrm{H}} \\
{\left[10^{22} \mathrm{~cm}^{-2}\right]}\end{array}$ & $\begin{array}{c}\text { PN Total } \\
\text { counts }\end{array}$ & $\begin{array}{c}\text { PN hard } \\
\text { counts }\end{array}$ & $\begin{array}{c}\text { Relative } \\
\text { hardness }^{a}\end{array}$ \\
\hline WR 1 & 0.3 & 1482 & $5(5-10 \mathrm{keV})$ & $0.3 \pm 0.15 \%$ \\
WR 6 & 0.4 & 2470 & $41(5-10 \mathrm{keV})$ & $1.7 \pm 0.3 \%$ \\
WR 110 & 1.1 & 1916 & $52(6-10 \mathrm{keV})$ & $2.7 \pm 0.4 \%$ \\
\hline
\end{tabular}

${ }^{a}$ Relative hardness is the ratio of the hard counts to the total counts in the PN instrument.

(Daltabuit \& Cox 1972). Consequently, the observed luminosity ratio across the edge can be used to infer either the sulfur abundance or the total photoabsorptive cross-section below the S-edge. Models of stellar evolution for massive stars indicate that WN stars are largely He-rich, $\mathrm{H}$ deficient star with nonsolar CNO abundances, but that the heavier metals, such as Ne, $\mathrm{Mg}, \mathrm{S}, \mathrm{Si}, \mathrm{Fe}$, should all be solar. These predictions seem to be on good observational footing (e.g., Morris et al. 2000). We feel that it is more reasonable to assume the sulfur abundance, for which we use $\mathcal{A}_{\mathrm{S}}=4.8 \times 10^{-5}$ from van der Hucht et al.

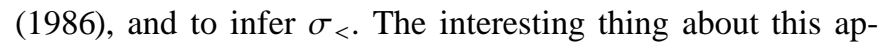
proach is that $\sigma_{<}$is primarily sensitive to the He absorption and the CNO abundances which have edges shortward of S.

From our model fitting, we can infer a luminosity ratio of $L_{<} / L_{>} \approx 6$, but it can range from as low as 2 to as high as 16. Unfortunately, its value is not well-constrained because the counts at these energies are low. Inserting the nominal value into Eq. (8) yields an estimate for the total wind cross-section short of the S-edge of $\sigma_{<} \approx 1.1 \times 10^{-24} \mathrm{~cm}^{-2}$. Using the parameters of Table 1 for the properties of WR 1 , this observationally inferred cross-section can be used to estimate the value of $r_{1}$ below the S-edge. Assuming a standard wind velocity law with $\beta=1$, we obtain $r_{1} \approx 3 R_{*}$. This is significantly below the expected value, by a factor of 10 . It could mean that the N-abundance of WR 1 has been overestimated. If lower, the photoabsorption cross-section for the model would also be lower; however, we must caution here as before that the range of acceptable values for the luminosity drop is broad. As a general point, it appears that the detection of drops in emission across known edge locations can be an important diagnostic of the metal abundances for the wind absorbing component.

\subsection{Hard emission}

The fact that all three putatively single WN stars - WR 1, WR 6, and WR 110 - show such similar X-ray spectra is somewhat gratifying, since it parallels trends observed among the O stars. But in the same way that the new X-ray data for O stars have spawned new questions, so the X-ray spectra of the WN stars are providing important and somewhat perplexing clues about the structure of WR winds. Both WR 6 and WR 110 exhibit significant hard emission beyond $4 \mathrm{keV}$. This hard component is essentially not seen in WR 1 .

We base our conclusion on the data appearing in Table 4. Listed there are the interstellar column density, total counts detected in the PN instrument, the detected hard counts, and the ratio of the two ("relative hardness"). The stars are listed in 
order of increasing interstellar column density. Above a few $\mathrm{keV}$, the ISM absorption is negligible; however, it is very important for the amount of soft emission that we measure at the Earth, since the bulk of the total PN counts come from the modest energies of about $1 \mathrm{keV}$.

Now in forming the relative hardness, one anticipates that if the sources are intrinsically similar, the ratios will grow with increasing values of $N_{\mathrm{H}}$ because more soft emission is absorbed, hence the spectrum becomes relatively harder. Indeed, this is the trend of the data. However, the interstellar column to WR 1 and WR 6 are very nearly the same, and yet the relative hardness of the X-ray spectrum for WR 6 is almost $6 \times$ greater. It is not unlikely that the 5 hard counts observed from WR 1 are consistent with a random Poissonian fluctuation in the average background level of 2 counts.

Consequently, we find no evidence for hard emission intrinsic to WR 1, which stands in opposition to the clear detections for WR 6 and WR 110. This conclusion is somewhat bolstered by the fact that Skinner et al. (2002a,b) find the hot component in WR 6 and WR 110 to be around $3 \mathrm{keV}$ or higher, whereas the hot component for WR 1 is closer to $2 \mathrm{keV}$.

Skinner et al. (2002a,b) argue that the hard emission in WR 6 and WR 110, given the lack of other evidence for binarity, may be a signature of the WR winds colliding onto non-degenerate and otherwise unseen stellar companions. In fact, it is interesting to consider the hardness of WR 25, a WN6 star that has long been known to be anomalously X-ray bright indicating that it may be a colliding wind binary system (Pollock 1987, 1991), although no companion has been directly detected so far (Moffat 1978; Conti et al. 1979; Drissen et al. 1992; van der Hucht 2001). Raassen et al. (2003) have reported on XMM observations of this source. A relative hardness of about $3 \%$ for the PN results from taking the count rate above $5 \mathrm{keV}$ and dividing by the total count rate (Antokhin, private comm.). Raassen et al. quote a value of $E(B-V)=0.53$, implying a $\mathrm{H}$ column density of $0.2 \times 10^{22} \mathrm{~cm}^{-2}$, which is even lower than for WR 1. So WR 25 is a fairly hard source, which is often taken as suggestive of binarity. It would thus stand to reason that the much lower spectral hardness in WR 1 suggests the star is single, or at least not a close binary. Consequently, the XMM-Newton observation of WR 1 may represent the first time that a detailed X-ray spectrum has been measured in a WR star that isolates the wind contribution alone, in contrast to $\mathrm{X}$-rays from wind accretion or colliding winds.

\section{Conclusion}

We have presented XMM-Newton observations of the nitrogen-rich Wolf-Rayet star WR 1. Using the EPIC suite of instruments - PN, MOS1, and MOS2 - we obtained a low resolution spectral shape characterized primarily by strong ISM absorption below $0.5 \mathrm{keV}$, peak emission around $1 \mathrm{keV}$, and some harder emission up to $4 \mathrm{keV}$. Although individual lines cannot be resolved, there are significant "bump" features that coincide with lines known to be strong in other hot stars, namely MgXI, SiXIII, and possibly SXV. There is some hint that the $\mathrm{H}$ and He-like nitrogen lines may be present, but these, being located in the strongly absorbed portion of the soft band, are highly uncertain. More interesting is the possibility that a S-edge near $2.6 \mathrm{keV}$ is detected. Edges at lower energies in mediumresolution spectra are difficult to detect owing to the preponderance of emisson lines that tend to "wash out" the absorption drop, and edges at higher energies are hard to see owing to the much lower fluxes and noisier data. The S-edge is ideally situated, being at energies above the forest of Fe lines but not so high as to appear in the faint tail of the spectrum.

In stark contrast to WR 6 and WR 110 that have also been observed with XMM-Newton (Skinner et al. 2002a,b), WR 1 does not appear to have a significant hard component above $4 \mathrm{keV}$ for our exposure of $7000 \mathrm{~s}$. For WR 6 and WR 110, the hard emission might plausibly be understood as wind accretion onto a otherwise undetected and non-degenerate companions. The lack of such hard emission from WR 1 may imply that this WR star is indeed single, so that for the first time, we may genuinely have isolated the X-ray emission from a single WR star wind.

There is an important point to be made in relation to the $\mathrm{S}$-edge and the hard emission. We claim to have detected the S-edge in the spectrum of WR 1 , but this edge is not seen in WR 6 or WR 110. If wind accretion onto a companion is invoked to explain the hard component observed in those stars, and if that emission contribution dominates over contributions from shocks in the respective WR winds, then no S-edge would be expected if the binary separation is greater than the $r_{1}$ level at $2.6 \mathrm{keV}$. In the case of WR $1, r_{1}$ is approximately $0.2 \mathrm{AU}$ at the S-edge, and should be similar for WR 6 and WR 110.

In our opinion, a key objective in the near future will be to obtain resolved emission profiles of strong unblended X-ray lines from these WR stars. A good starting point would be WR 1 , since it seems to be a single star. If the X-ray emission truly emerges from hot gas distributed throughout the wind flow at large radius, where the wind flow is in constant expansion, then the line profile shapes should either be "flattopped" (consistent with no wind absorption and suggestive of a large scale hot bubble) or asymmetric with blueshifted peak emission (consistent with hot gas distributed throughout the wind according to the prediction of Ignace (2001) based on the standard wind-shock paradigm). If relatively narrow, unshifted emission lines were to be observed, as has been the case in some OB stars, this might imply an alternate mechanism for the $\mathrm{X}$-ray production (e.g., magnetic fields) or some unusual structuring of the wind flow that would invalidate how the X-ray production and/or the warm absorption by the stellar wind is normally treated. Either case might present a serious challenge to our understanding of WR winds and could have ramifications for how high mass-loss winds are driven out from these stars.

Acknowledgements. We acknowledge many helpful discussions with J. Cassinelli. We especially thank W.-R. Hamann for providing atmosphere models of WR 1, and I. Antokhin for guidance on using XMMNewton's SAS. We appreciate assistance given by G. Lamer on some technical points related to the SAS. Our thanks also to an anonymous referee. This study was initiated at the University of Glasgow and was supported by a PPARC Grant (RI, JCB) and a NATO Fellowship (LMO). RI acknowledges support for this research from a NASA grant 
(NAG5-12557), and LMO acknowledges support from a Deutsche Forschungsgemeinschaft grant (Fe 573/1-1).

\section{References}

Baum, E., Hamann, W.-R., Koesterke, L., \& Wessolowski, U. 1992, A\&A, 266, 402

Berghöfer, T. W., Schmitt, J. H. M. M., Danner, R., \& Cassinelli, J. P. 1997, A\&A, 322, 167

Cassinelli, J. P., Miller, N. A., Waldron, W. L., MacFarlane, J. J., \& Cohen, D. H. 2001, ApJ, 554, L55

Cohen, D. H., Cassinelli, J. P., \& MacFarlane, J. J. 1997, ApJ, 487, 867

Cohen, D. H., de Messieres, G. E., MacFarlane, J. J., et al. 2003, ApJ, 586,495

Cooper, R. G., \& Owocki, S. P. 1994, Ap\&SS, 221, 427

Conti, P. S., Niemela, V. S., \& Walborn, N. R. 1979, ApJ, 228, 206

Craig, I. J. D., \& Brown, J. C. 1986, Inverse Problems in Astronomy (Bristol: Hilger)

Daltabuit, E., \& Cox, D. P. 1972, ApJ, 177, 855

Donati, J.-F., Babel, J., Harries, T. J., et al. 2002, MNRAS, 333, 55

Drissen, L., Robert, C., \& Moffat, A. F. J. 1992, ApJ, 386, 288

Feldmeier, A., Puls, J., \& Pauldrach, A. W. A. 1997, A\&A, 322, 878

Feldmeier, A., Oskinova, L., \& Hamann, W.-R. 2003, A\&A, 403, 217

Gayley, K. G., \& Owocki, S. P. 1995, ApJ, 446, 801

Groenewegen, M. A. T., \& Lamers, H. J. G. L. M. 1989, A\&AS, 79, 359

Howk, J. C., Cassinelli, J. P., Bjorkman, J. E., \& Lamers, H. J. G. L. M. 2000, ApJ, 534, 348

van der Hucht, K. A., Cassinelli, J. P., \& Williams, P. M. 1986, A\&A, 168,111

van der Hucht, K. A. 2001, NewAR, 45, 135

Ignace, R., Oskinova, L. M., \& Foullon, C. 2000, MNRAS, 318, 214

Ignace, R. 2001, ApJ, 549, L119

Ignace, R., \& Gayley, K. G. 2002, ApJ, 568, 954

Kaastra, J. S. 1992, An X-ray spectral code for optically thin plasmas, Internal SRON-Leiden Report
Kahn, S. M., Leutenegger, M. A., Cottam, J., et al. 2001, A\&A, 365, L312

Kramer, R., Cohen, D. H., \& Owocki, S. P. 2003, ApJ, 593, 532

Lucy, L. B., \& White, R. L. 1980, ApJ, 241, 300

Lucy, L. B. 1982, ApJ, 255, 286

MacFarlane, J. J., Cassinelli, J. P., Welsh, B. Y., et al. 1991, ApJ, 380, 564

Mewe, R., Gronenschild, E. H. B. M., \& van den Oord, G. H. J. 1985, A\&AS, 62, 197

Miller, N. A., Cassinelli, J. P., Waldron, W. L., MacFarlane, J. J., \& Cohen, D. H. 2002, ApJ, 577, 951

Moffat, A. F. J. 1978, A\&A, 68, 41

Morris, P. W., van der Hucht, K. A., Crowther, P. A., et al. 2000, A\&A, 353,624

Niedzielski, A. 2000, A\&A, 357, 581

Oskinova, L. M., Ignace, R., Hamann, W.-R., Pollock, A. M. T., \& Brown, J. C. 2003, A\&A, 402, 755

Owocki, S. P., Castor, J. I., \& Rybicki, G. B. 1988, ApJ, 335, 914

Owocki, S. P., \& Cohen, D. H. 1999, ApJ, 520, 833

Owocki, S. P., \& Cohen, D. H. 2001, ApJ, 559, 1108

Pollock, A. M. T. 1987, ApJ, 320, 283

Pollock, A. M. T. 1991, in Wolf-Rayet Stars and Interrelations with Other Massive Stars in Galaxies, ed. K. A. van der Hucht, \& B. Hidayat (Dordrecht: Kluwer), IAU Symp., 143, 105

Pollock, A. M. T., Haberl, F., \& Corcoran, M. F. 1995, in WolfRayet Stars: Binaries, Colliding Winds, Evolution, ed. K. A. van der Hucht, \& P. M. Williams (Dordrecht: Kluwer), IAU Symp., 163, 191

Raassen, A. J. J., van der Hucht, K. A., Mewe, R., et al. 2003, A\&A, 402,653

Schulz, N. S., Canizares, C. R., Huenemoerder, D., \& Lee, J. 2000, ApJ, 545, L135

Seward, F. D., Forman, W. R., Giaconni, R., et al. 1979, ApJ, 234, L55

Skinner, S. L., Zhekov, S. A., Güdel, M., \& Schmutz, W. 2002a, ApJ, 572,477

Skinner, S. L., Zhekov, S. A., Güdel, M., \& Schmutz, W. 2002b, ApJ, 579,764

Waldron, W. L., \& Cassinelli, J. P. 2001, ApJ, 548, L45 